\title{
Using Parsimony to Guide Maximum Likelihood Searches
}

Hyrum Carroll

hyrumcarroll@gmail.com

Mark J. Clement

clement@cs.byu.edu

Timothy O'Connor

Quinn O. Snell

Kenneth Sundberg

Follow this and additional works at: https://scholarsarchive.byu.edu/facpub

Part of the Computer Sciences Commons

\section{Original Publication Citation}

Using Parsimony to Guide Maximum Likelihood Searches, Kenneth Sundberg, Timothy O'Connor, Hyrum Carroll, Mark Clement, and Quinn Snell, IEEE Symposium on Biolnformatics and BioEngineering (BIBE), Washington, D.C., October 27, pp 774-779.

\section{BYU ScholarsArchive Citation}

Carroll, Hyrum; Clement, Mark J.; O'Connor, Timothy; Snell, Quinn O.; and Sundberg, Kenneth, "Using Parsimony to Guide Maximum Likelihood Searches" (2007). Faculty Publications. 230.

https://scholarsarchive.byu.edu/facpub/230 accepted for inclusion in Faculty Publications by an authorized administrator of BYU ScholarsArchive. For more information, please contact ellen_amatangelo@byu.edu. 


\title{
Using Parsimony to Guide Maximum Likelihood Searches
}

\author{
Kenneth Sundberg*, Timothy O'Connor ${ }^{4}$ Hynum Carroll $^{*}$, Mark Clement*, and Quinn Snell ${ }^{*}$ \\ * Computer Science Department. \\ Brigham Young University \\ Provo, Utah 84606 \\ Email kasundberg@gmail.com, ho@ cs byu.edu, clement@ cs.byu.edu, snell@cs.byu.edu \\ Integrative Biology Deparment \\ Brigham Young University \\ Provo, Utah 84606 \\ Email: timothydoconnor@gmail.com
}

\begin{abstract}
Abtrat-The performance of maximum likelihood searches can be booted by using the nost parsimonious tree as a staring point for the search. The time spent in performing the parsimony search to find this starting tree is insignificant compared to the time spent in the maximum likelihood search, leading to an overall gain in search time. These parsimony boosted maximum likelhood searches lead to lopologies with scores statsically similar to the unbosted searches, but in less time.
\end{abstract}

\section{INTRODUCTION}

There are two common methods for inferring phylogenies from multiple alignment data, maximum parsimony [2] (MP) and maximum likelihood [10] (ML). The search problem for both methods is known to be NP-Hard [G] with parsimony known to also be NP-Complete [7], however the scoring of individual topologies during the search is quite different. The parsimony score of a topology can be computed in linear time with respect to the number of nodes in the topology, whereas the best known ML computations run in exponential time with respect to the number of nodes. As a result MP searches are much faster than ML searches.

Unfortunately parsimony has a strong bias toward long branch attraction, and can lead to positively misleading topologies [9]. Maximum likelihood also models more of the biology, including probabilities and estimates of branch lengths, information that can be required by other methods of phylogenetic analysis. As a result of the flaws of parsimony and the additional infonnation provided by maximum likelihood, maximum likelihood is preferred by many researchers.

\section{DATA SETS}

For this work we used three types of alignment data sets: four laxa synthetic data, small real data sets and large data sets.

\section{A. Synthetic Alignments}

The four taxa synthetic data sets were used to establish areas of general concordance between ML and MP. To produce them we used the program Dawg [5] under a General Time Reversible (GTR) [26], [17], [22] model of evolution. We

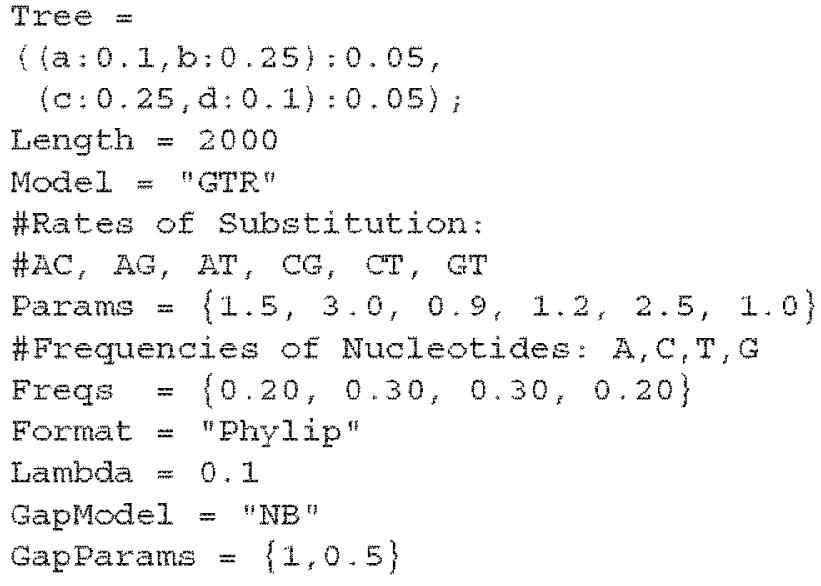

Fig. 1. Typical file given to Dawg (Felichstein zone)

modeled the parameters according to the examples included with the program and explored a range of branch lengths as seen in Figure 1. The lambda value of 0.1 was used for the indel evolution rate and can be interpreted as one indel for every ten substitutions. The sequence length was increased to 2000 as this gives a reasonably sized sequence to allow for the expected value of any simulation to be seen. We ran two types of trees, a Felsenstein topology and a Farris topology, see Figure 2. Dawg generated data sets for trees under both topologies where the $a$ and $\beta$ branch lengths were varied from a branch length of 0.1 to 4.05 incremented by 0.05 . A branch length of one is interpreted to mean that each site is expected to have one substitution from the internal node under the GTR definition of branch length. For each permutation of $\alpha$ and $\beta$ branch lengths we ran a hundred replicates to get a percentage of matches between the two methods. This created a total of $1,280,000$ data sets. In addition, this entire procedure was repeated with the the model changed to Jukes-Cantor (JC) [15], creating an additional $1,280,000$ data sets. 


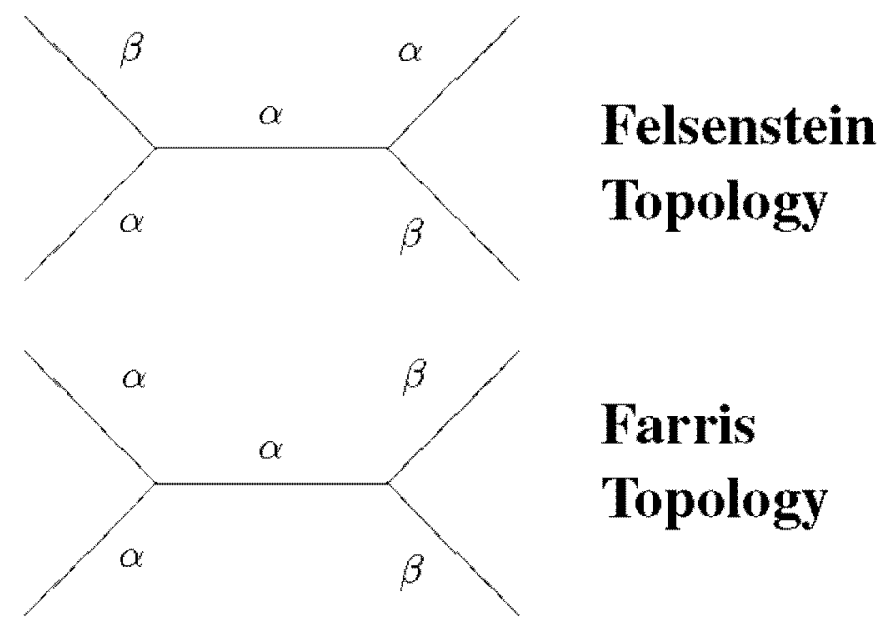

Fig. 2. The Felsenstein and Farris Tress

\section{B. Small Real Alignment Data Sets}

We used all the data sets whin nine, ten, and eleven toxa from the mdse $100 \mathrm{~s}$ version of the benchmarks published by Carroll et al. [3]. These 279 data sets are protein-coding DNA algnments, detived from reference amino acid alignments [27], [20], [8], [19]. The small real ahgmments were used to perform exhanstive MP and ML searches.

\section{Large Data Sers}

We also used several large data sets provided by Stamatakis (http/howwweph.ch/stamatak/ndex-Datelen/Page443.hm). These data sets include seven ral alignments ranging from 101 to 500 taxa and 10 simulated data sets, each with 4000 taxa and 2000 base pairs. These data sets are nsed to evaluate our heuristic on large data sets using RAXML [24].

\section{CORRELATIONS}

Both parsinony and naximum likelhood methods try to extract the evolutionary signal on of a mutiple sequence alignment. Though they do so in very different ways it is reasonable to expect a lair correspondence between the lwo methods.

\section{A. Areas of General Concowdance of ML and MP}

There has been a large inquiry into the relationship of $\mathrm{ML}$ and MP from the perspective of accuracy and strength of methods [23], [13], [14], [16] but the comparison has never taken the view of how well one estimates the other. Here we have used simulated data to elucidate this relationship. To do so we focmed on the standard four taxa case and compared the performance of both methods under a Felsenstein topology and a Farris topology.

This topology as seen in Figure 2 is a four taxa tree with non-sibling branches that have branch length $\beta$ and all other branches with lengh $\alpha$. The second tree in Figure 2 shows a Fanis topology where the two $\beta$ length branches are siblings.

The Felsenstein zone 191 can be loosely described as the subspace of all Felsenstein topology branch lengths where

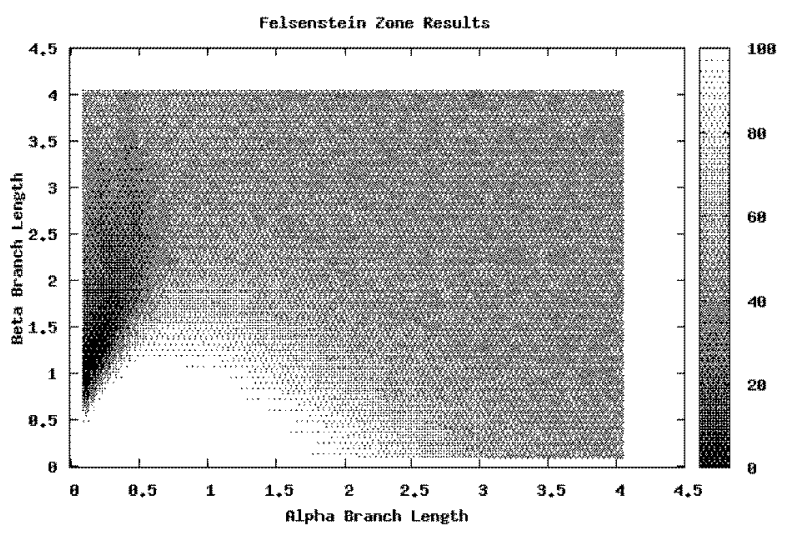

Fig. 3. Chart showing the percentape of datasets of a given the Felsenstem Topology where Maxinum Parsinony and Maxinum Likethood predinted the same optimal topology.

parsinony will predict a tree topology that is incorrect. Later work went on to show that ML performs well on Felsenstein topologies, holnding those that lie in the Felsensteio zone and reasonably well on Fanis topologies. Aso shown was that MP fatled in certain areas of branch lengths in the Felsenstein topology but excelled at the Farris topology. [23], [13], [14], $[16]$

Parsimony, has a strong bias towand placing longer branches as siblings. This bias gives it an advantage when longer branches are 'buly' siblings and a disadvantage when the "rue" tree has non-sibling long branches.

To illustrate this we scored simulated alignments, as described in section II-A and compared the topological result produced from ach method, the resuls are shown in Figures 3 and 4 . These gaphs show the percentage of time each method chose the same tree of the three possible trees.

As is clearly shown, one area in the Felsenstein topology space (the Fulsenstein zone), as predicted by previous studies, does not correlate well forming the dark parabolic stucture in Figure 3. This is due to the long branch atraction problem (LBA). From the pexpective of parsimony as an estimator of likelihood topology, this should not be a problem becanse the mumber of long branches in a large data set are usually few and would only vary the topology by a few branches that are incorrect according to likelhood.

By ruming a lkelihood seach based on the best parsinomy tree we can save time by getting the majonty of branches correct and leaving likelibood to figure out the incorrect branches. This saves on the cost of using likelihood to search the whole tree space but lets it fne tune after the majority of bad trees are ruled out by parsimony. We are not trying to use pasimony as a function to predict ML but as a method to boost the search pass the large number of trees that both procedures predict are incorrect.

Most trees are assmmed to be within the aren where both 


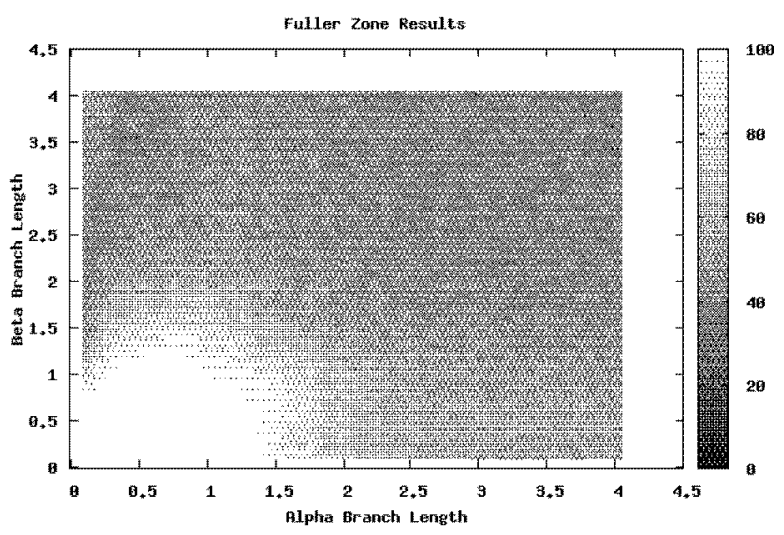

Fig. 4. Chart showing the perwentage of datasets of a twen true Farris Topology where Maximum Parsimony and Maxmum Lilohihow predicted the same optinal topology.

methods are completely consistent, the light colored areas of Figures 3 and 4 . In the gray areas of the figures the methods are doing no better than randomly gnessing. This is to be expected as this occurs where there is an expected rate of 2 or more substitutions per site. which causes a large loss of phylogenetic signal. As expected, the major limitation to the concordance between ML and MP are those topologies that lie within the Felsenstein zone.

Each tree was scored using the phylogenetics program PAUP* [25] under both MP and ML. We derived the results shown in Figures 3 and 4 using the defaults of PAUP* for parsimony and likelihood. The model for likelihood used was HKY [12],

This is an under-parametrization of the base model, GTR, used by Dawg to generate the data. The nse of this nnderparametriation, as HKY is a more simplified nodel compared to GTR, was used because it allowed for a miversal model for all data sets withou having to calculate the optimum parameters or model for each data set. To further explore the effects of this assumption we reran the analysis using a base model of JC in Dawg to see if there was a change in the results. In this case the use of $\mathrm{HKY}^{\prime}$ in the like lihood evaluation would be an over-parametrization of the the data sets model. We found no particulanly different results (data not shown).

\section{B. Gap State}

When using parsimony as a hemistic for likelhood the gap mode used for the parsinony score is critical for good correspondence. Treating the gaps in an alignment as a new state creates large variances in the parsimony scores, depending on the extent to which the gaps line up. One solution when using parsimony as a precursor to a maximun likelihood search to set the gap mode to missing to avod this difficulty. Figures 5 and 6 show the difference in correspondence between the two methods. Both figures are a plot of an exhaustive search

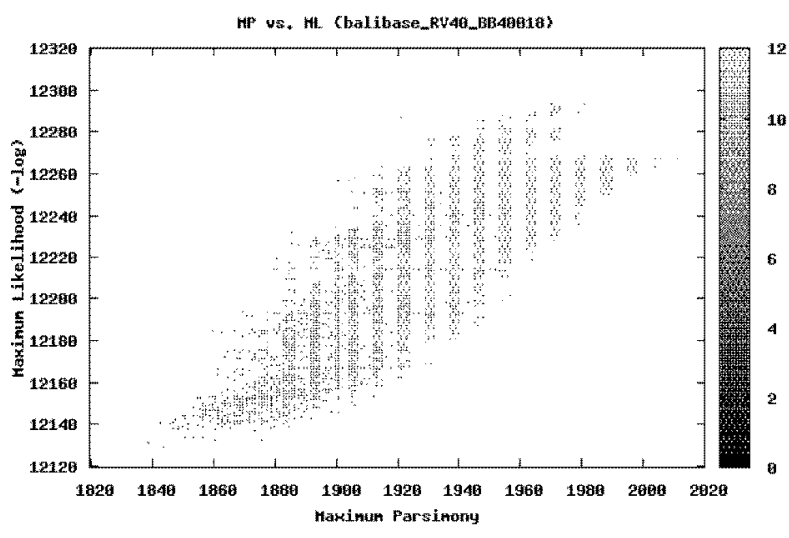

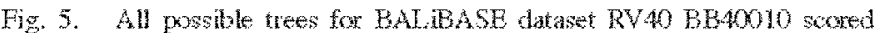
with parsimony (GapMods = missing) and maximum likelihood (HKY under

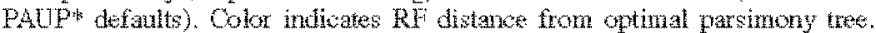
Wote that the optimal tre under belihood is a small RF distance from the otimal parsimony tres.

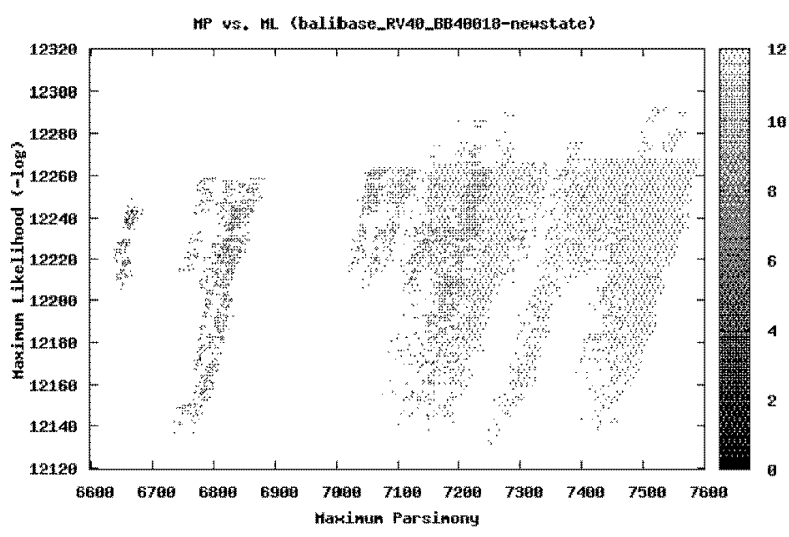

Fig. 6. All porible trees for BALiBASE dataset RY40 BBA610 acored with parsinony (GapMode - new state) and maximum likelihood (HKY under PAUP* defauls). Color indicates RF distance from optimal pasimory tres. Note that the optimal tree ander likelihood is a large RF distance from the optimal garsinony tre.

through one of the BAliBASE [27] data sets (RV40 BB40010). Every possible topology was scored by both parsimony and maximum likelibood, and then plotted The color of each point indicates the topological distance, (measured using Robinson Foulds [21], from the most parsimonious topology. In both cases maximum likelhood was scored using the HKY method. These results are representative of the other data sets.

\section{Correlation Between The Most Likely and The Most Pur- simonious Topology}

While the correspondence between MP and ML methods exists it does not preserve a partial order between topologies. 


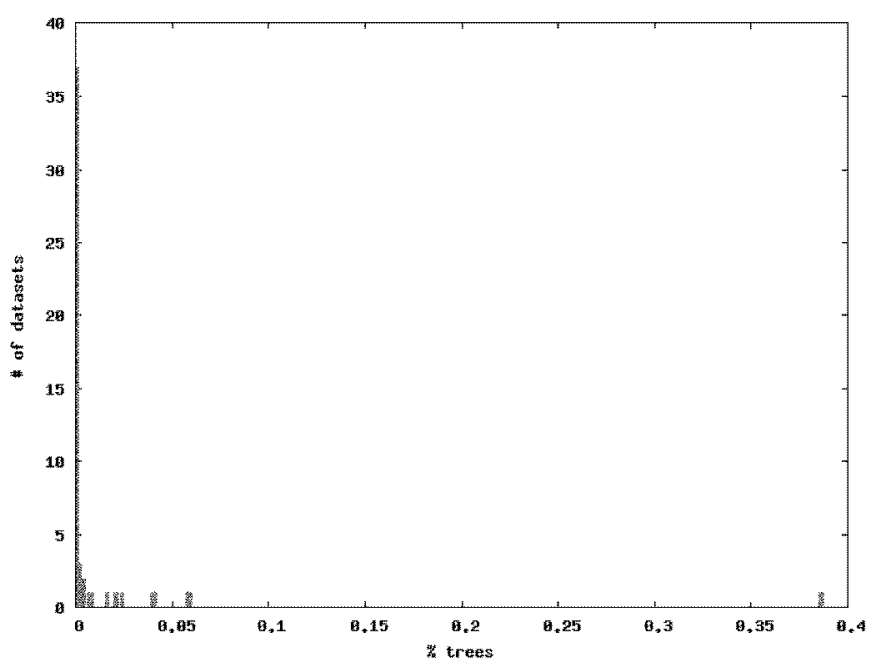

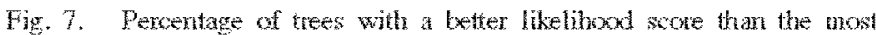
parsinonious tres

It is therefore not possible to direclly substitute one method for the other. However it is possible to use parimony to aid a maximm likelhood seard. Figure 5 shows that as parsimony score decreases, the negative log naximum likehiood score generaly decreases also This trend also generalizes to the other data sets studied.

In all but two of the nearly 300 real dats sets, less than 70 possible topologies had a better likelhood score than the most parsimonious topology for that dataset. It is clear that in the majority of cases, parsimony eliminates most of the incorrect topologies. Fignre 7 is a histogram of data sets grouped by the percentage of trees with betur ikelihood scotes than the best parsimony tree. Note that 37 datasets fell into the $0 \%$ tress bucket. Tor the 9 taxa case, from which these data sets have been drawn $0.05 \%$ corresponds $106 \%$ trees.

Furthemone the likelhood scote of the best parsimony tres is oflen very comparable to the likelihood score of the best maximum likelihood tree. Figure 8 shows the relative error of the best parsinony tree, taking the best maximum likelibood score as the tue valne.

\section{PARSIMONY HEURISTIC}

Not only do likelihood and parimony generally improve together, the most likely and the most parsimonions trees are often very close topologically. The major dimcnly with parsimony tnethods is long branch atraction [1]. This problem. causes two long branches to be incorrectly made siblings in the inal topology. This topological change is however conectable with a very small mumber of TBR swaps. A simple hemistio that can be appled to many existing phylogenetic programs is to perform a parsimony search and use the result as the seed tree for a maxmum likelihood search. This maximum likelhood search can be further limited to trees within a amall TBR distance of the nost parsimonious tres.

The idea of using parsimony to improve likelinood searches is not entirely new. RAXML uses a step-wise maximum

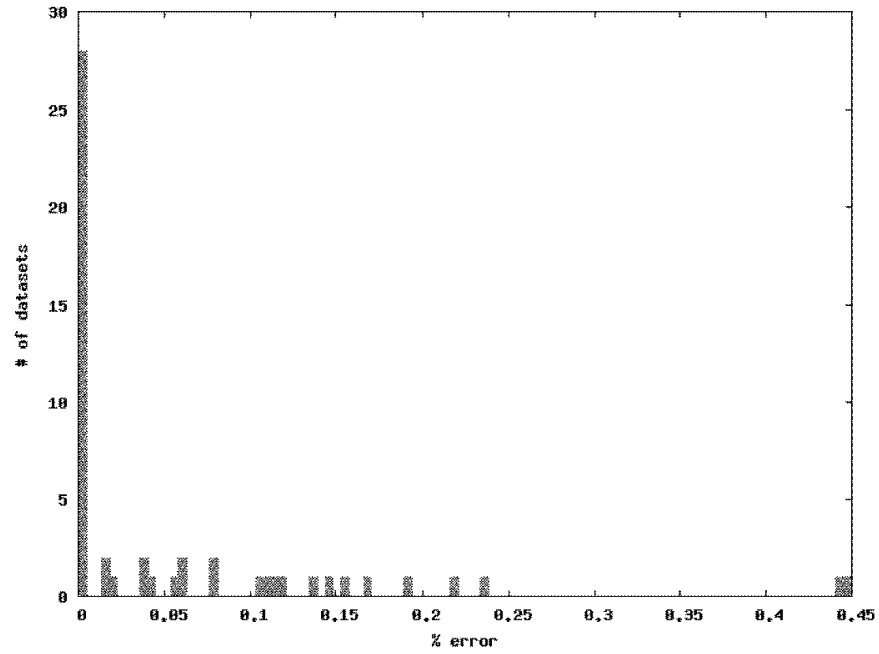

Fig. Relative exror in ML sore of MP tre

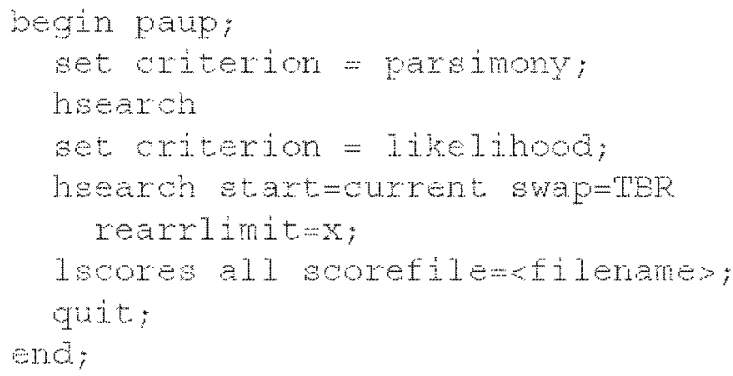

Fig. 9. PAUP" block to perform heuratic search

parsimony tree as its stating tree [24]. This is a step in the right direction but perfoming a complete parsimony search further improves perfomance of this search.

\section{A. Improwing PAUP*}

Our heuristic is very easily implemented in PAUP*: PAUP: already has the machinery to perfom both parsimony and maximum likelihood searches. The heuristic can be expressed in a PAUP block, as shown in Figure 9. As parsimony gives ns a tree that is lopologically close to the desired tree, we himit the length of the maximum likelihood search, gaining further savings in time. The $x$ used as the rearrlamit was set to the mumber of trees within one "T $\mathrm{BR}$ swap.

We acheved identical scores on the majonity of data sets using this method. Figure 10 shows a histogram of the differences in the scores of the final tree. It is not statistically significant that occasionaly the heuristic outperfoms the standard PAUP* search, nor is it signifont that the standard PAUP: search occasionally produces a better score than the heuristic.

The nse of the beutistic does improve search times significantly. As shown in Figure 11, the run time was on average improved by $25 \%$. In a handful of the data sets, parsimony was not a good indicator of likelibood and the search took longer using the heuristic than it would have otherwise. All 


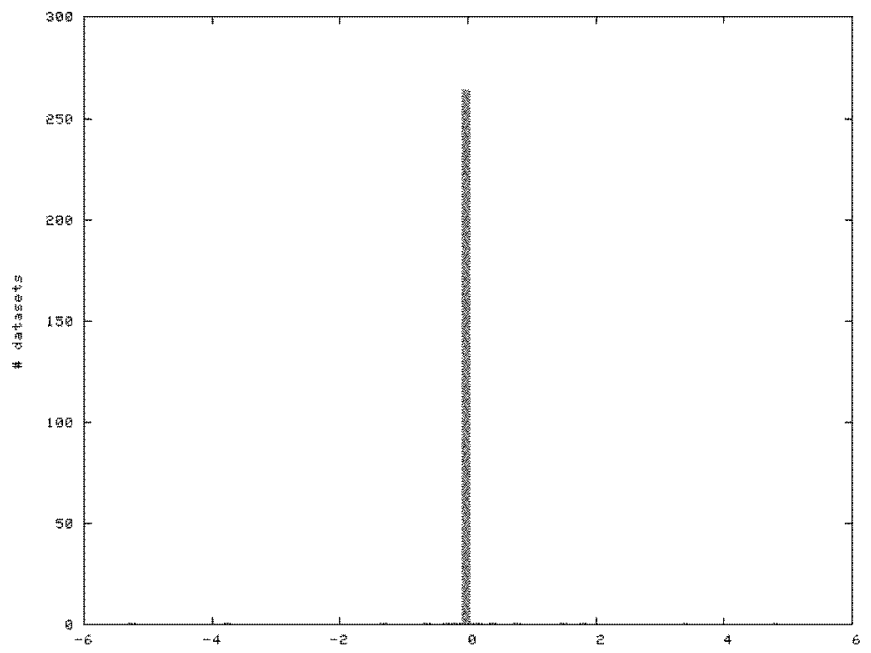

Fig. 10. Sore differences between PAUP: with ard without the Parsinny Heuriati:

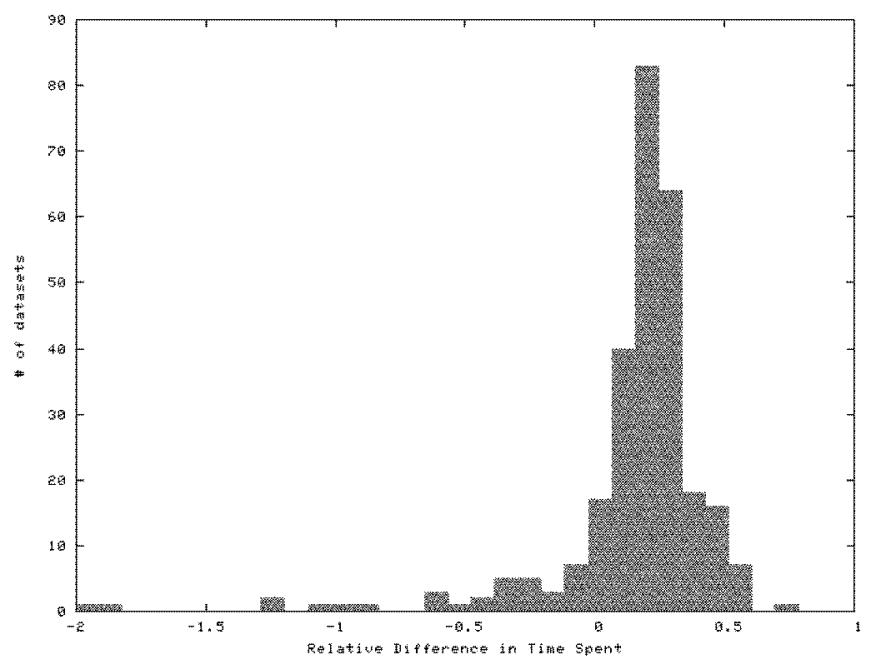

Fig. 11. Running time inprowent fron starting with a parsinony search in PAUP*

times are the average of tive runs.

\section{B. limprowing RAxML}

Due to the size of the large data sels, exhaustive searching is not feasible. RAXML is a phylogenetic search program that can perform maximum likelihood searches on large data sets [24]. One of the heuristics it uses is to start the ML searching with a stepwise maximum parsimony iree. We took a slightly different approach to applying our heuristic to RAxML. First, we heuristically searched under MP, and then started the ML, search in RAXML with the most parsimonious tree found. To search with MP, we used TNT, Tree Analysis Using New Technology [1]1, [18]. In other analysis that we ve performed, TNT out performs PAUP* in terns of time and parsinony score [4]. We used two TNT search strategies per each date set: 1) sectorial-search and smult and 2) TBR. The most parsimonious tree found with TNT was used to start a

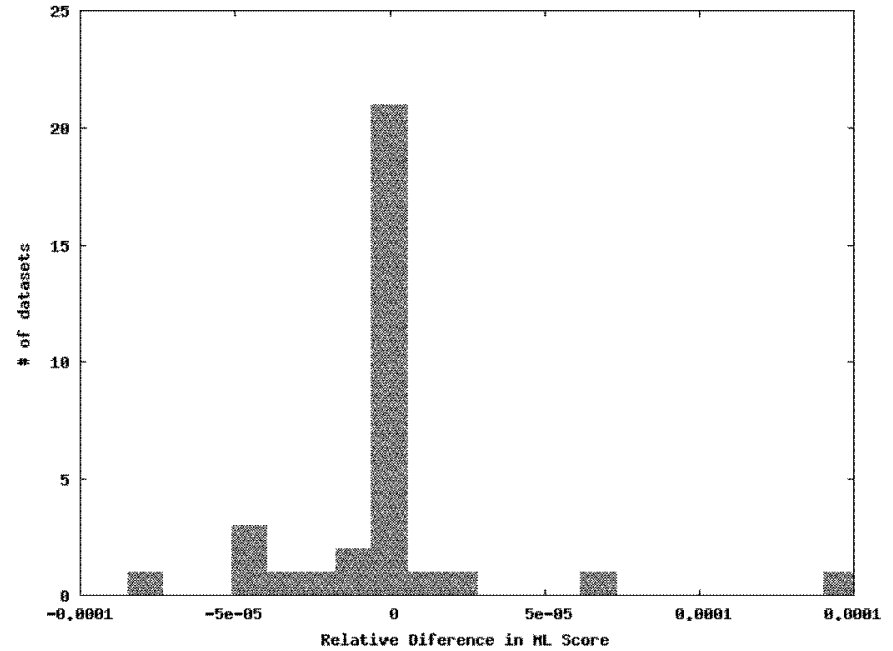

Fig. 12. Swore differences between RAMML with and without using TNT

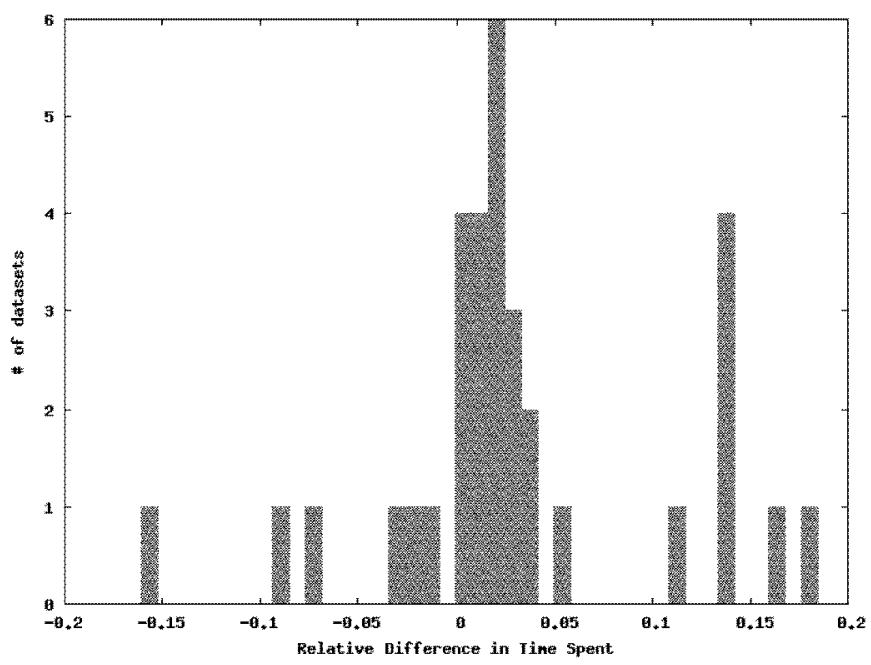

Fig. 13. Execute tims improvements using RAxkd and TNT versus just RAXML

GTR-GAMMA search with RAxML. The relative difference in scores is shown in Figure 12. Again the scores are not statistically different, but there is more variability than with the PAup: scores.

The execution time improvements are shown in Figure 13. There are two circumstances which are probably cansing this reduction in the performance of the heuristic. First, RAxML by default starts its search with a step-wise maximum parsimony tree. This tree already has a reasonable parsimony score, so there is not as mach benefit to be gained by finding a more parsimonions tree. Another difference is that RAxML does not allow us to limit the time spent in the likelhood search, which does not allow us to take full advantage of the small topological differences between the maximum parsimony and maximum likelihood trees. 


\section{CONCLUSTONS}

Parsimony is correlated with likelhood in phylogenetic inference. In many data sets the predicted topologies are very close, if not identical. Maximum likelhood searches can be boosted using our parsimony heuristic in a manner that avoids the problems asscoiated with the Felsenstein Zone and parsimony while mantaining some of the speed of a parsimony search.

\section{REFERENCES}

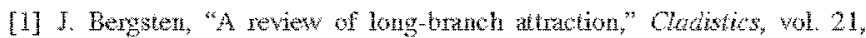
no. 2 , pp. $163-193,2005$.

2] J. Camin and R. Sokal, "A Mehod for Dedwing Branching Sequences in Phylogeny," Equation, vol, 19, no, 3, pp. $311 \ldots 96,1965$.

(3) H. Carnl, W. Becksead, T. OConno, M. Bboert, M. Clement, Q. Snell. and D. MoClellan, "DNA Reference AHgment Benchmats Based on Totiary stneture of thended proteins, in press with binin. formatics.

(4) H. Carol. M. Gement, Q. Snel, and K. Gradal, "Phylomenetic

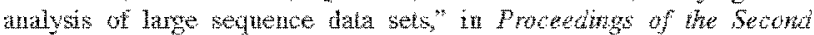
Fiotechnolog and Fionformatics Symposizm, 2005 , pp. $20-24$

[5] R. Curwright, "DNA assembly with gaps (Dawg): imuluting sequence cvolution," Bioifformaties, vol. $21,10.3, \mathrm{pp}, 31 \ldots 38,2005$.

[6] $\mathrm{B}$. Chor and "T. Tuller. "Maximam Hkelhood of evolutomary toes is ham, protedings of the oth Annat haternationd Conterence on Reseoreh in Complational Molectar Bivlogy (RECOMD 2005 , wol. $3500,0 p \cdot 296-310,2005$

(7) W. Day D, Jomson, and D. Sakof, "The computational omplexity of

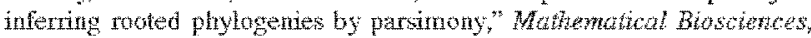
vol. 81, no. $3342,1.299,1986$.

[8] R. C. Edgar, "MUSCLE: muliple secuence algnment with high acuracy and high throughut," Nucleic Acids Resarats, vol. 32 , no. 5 , pp. $1792-1797$, mat 2004

19) 1. Holenetein, "Cases in which Parimony or compatibity Mothods Will to Positively Misleading," Systematic Loology, wol. 27, no. 4, po. $401-410,1978$.

[10] "Evolutomary Tres from DNA Sequetces: A Maximum Like-

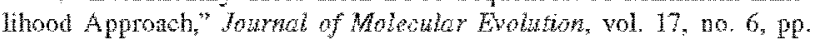
$368-376,1981$

111) P. Golobotf, S. Farris, and K. Nixon, "INT: Tree analysis nsing nev techology" htp:/wwwelabisties com/webththmi, 2001 .
[12] M. Hasegayd, H. Kishino, and T, Yano, "Dating of the fluman-aye spliting by a molecular clock of mitochondrial DNA," Joumal of Holecular Evolufion, vol. 22, no. 2, pp. 160 174, 1985.

[13] J. Huelsenbeck, "Perfomance of Phylogenetic Methods in Simulation," Systenatic Rotog, wol. 44, no, 1, pp. 17.48, 1995.

[14] J. Huelsenbek and D. Wills. "Sucess of Phylogenetic Methods in the

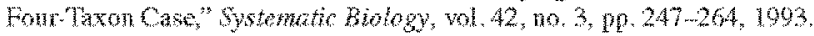

[15] T. Thkes and $\mathrm{C}$. Cantor, "Evoluton of proted molecules" Mommalion Frafew Metobolisw, vol. 3, pp. 21-132, 1969.

$116]$ M. Kuhner and J. Felsenstein, "A simulation comparison of phylogeny algonthms under equal and unequal evolutionary rates [published era tum appoar in Mol Biol Ewl 1995 May; 12 (3). 525]" Mol Bal Evol, พ०1. 11, no. 3 , pp. $459.468,1094$.

[17] C. Lanave, G. Preparata, C. Sacone, and $G$. Serio, "A new method for caloulating evolutionary substitntion mates," Jownat of Moleculor

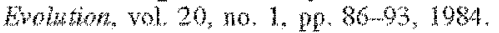

[18] R. Metwr and F. Al. "Sofwate Review. The rewest kil on the parsimany block: TNT (The analysis using new teolmology) " Systematio Ertomelogy, vol. 30 , no. 1, p. 179, 2005.

$119]$ C Ponting I. Schultz, F Muper, and P. Bork "SMART: identification and amnotation of domains from signaling and extracellular protein

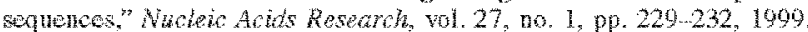

[20] G. P. S. Raghava, S. M. I. Senle, P. C. Audley, I. D. Barter, and

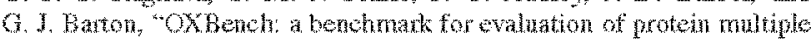

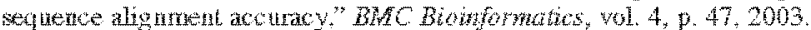

$211 \mathrm{D}$. Robinson and $\mathrm{I}$. Fonlds, "Comparison of Phylogenetic Tress" Materyatical Eirsetentes, wol 53, no, 1, pp. 131-147, 1981

221 F. Rodriguer, I. Oliver, A. Maxin and I. Medina "The genewal stochastic model of wucleotide substimtion." I Thear Roh vol. 142, no. 4, pp. $485-$ 501,1990 .

23] M. Siddall, "Sncess of Parsimony in the four "Taxon Case: Long Branch Repulsion by Likelhood in the Furis Zone," Cladistics, wol. 14, no. 3 , 10. $209.220,1998$

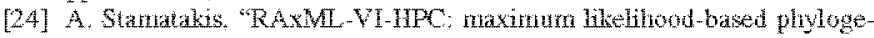
netic andyses with thonsands of taxa and mixed models", Bioinfomat i.s. wol. $22,110.21$, p. 2688,2006 .

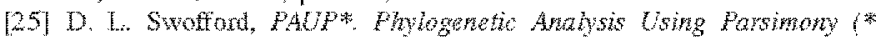

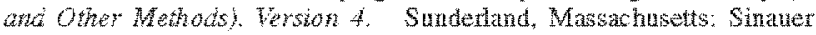
Associates, 2005 .

2615 . Tavare, "Some probabihatic and atatictical problems on the andysis

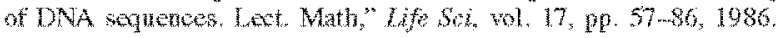

[27] I. D. Thompen, P. Kokh. R. Ripp, and O. Poch. "BALIBASE 3.0 latest

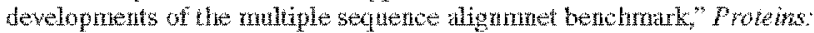

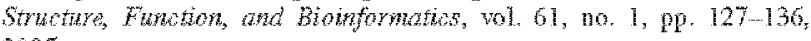
2005 . 